\title{
Characterization of Mouse Adult Testicular Macrophage Populations by Immunofluorescence Imaging and Flow Cytometry \\ Noushin Mossadegh-Keller ${ }^{1}$ and Michael H. Sieweke ${ }^{1,2,3, *}$
}

\begin{abstract}
${ }^{1}$ Aix Marseille Université, CNRS, INSERM, CIML, Marseille, France; ${ }^{2}$ Max-Delbrück-Centrum für Molekulare Medizin in der Helmholtzgemeinschaft (MDC), Robert-Rössle-Strasse 10, 13125 Berlin, Germany; ${ }^{3}$ CRTD-Center for Regenerative Therapies Dresden (CRTD), Technische Universität DresdenTU Dresden, Dresden, Germany

${ }^{*}$ For correspondence: Michael.sieweke@tu-dresden.de
\end{abstract}

[Abstract] Testicular macrophages (tMФ) are the most abundant immune cells residing in the testis, an immune-privileged organ. TMФ are known to exhibit different functions, such as protecting spermatozoa from auto-immune attack by producing immunosuppressive cytokines and trophic roles in supporting spermatogenesis and male sex hormone production. They also contribute to fetal testicular development. Recently, we characterized two distinct tMФ populations based on their morphology, localization, cell surface markers, and gene expression profiling. Here, we focus and describe in detail the phenotypical distinction of these two tMФ populations by fluorescence-activated cell sorting (FACS) using multicolor panel antibodies combining with high-resolution immunofluorescence (IF) imaging. These two techniques enable to classify two tMФ populations: interstitial tM $\Phi$ and peritubular $\mathrm{tM} \Phi$.

Keywords: Macrophage, Testis, FACS, IF, Spermatogenesis, Immune-privilege organ

[Background] The testis is the male reproductive organ where spermatogenesis and testosterone production occurs. tMФ might be considered as "guardians of fertility" by their immuno-suppressive function to assure the immune-privilege status of the testis and their trophic roles in spermatogenesis and male hormone production, but the distinct functions of these macrophage populations are only starting to be elucidated (Mossadegh-Keller and Sieweke, 2018). Tissue-resident macrophages exhibit tissue-specific functions and gene expression patterns depending of their organs of residency (Gentek et al., 2014; Lavin et al., 2014) but also share common core tissue-resident macrophage markers as F4/80, CD11b, CD64, M-CSFR and for some tissues CX3CR1 (Gordon, 2002; Gautier et al., 2012; Yona et al., 2013; Gentek et al., 2014). Recently, two testicular macrophages (tMФ) populations have been characterized using IF imaging and FACS (DeFalco et al., 2015; Mossadegh-Keller et al., 2017). Interstitial tMФ can be identified by microscopy by their rounded morphology and localization in the interstitial space of the testis in close contact with testosterone-producing Leydig cells (Smith and Walker, 2014; DeFalco et al., 2015; Mossadegh-Keller et al., 2017). In contrast, the peritubular IMФ exhibit an elongated morphology and surround the seminiferous tubules housing spermatogonial stem cells (SSC) (DeFalco et al., 2015; Mossadegh-Keller et al., 2017). Confirming previous observations (DeFalco et al., 2015), we observed that both $\mathrm{tM} \Phi$ were positive for CX3CR1 and F4/80 by IF. Interstitial tM $\Phi$ can be distinguished by the strong expression of M-CSFR from peritubular IM $\Phi$ that selectively express high 
levels of MHCII (Figure 2) (Mossadegh-Keller et al., 2017). We further analyzed in depth the interstitial and peritubular TMФ populations by FACS. We established an extended antibody panel going beyond previous protocols (DeFalco et al., 2015), excluding monocytes and dendritic cells and including CD64 as a key tissue-resident macrophage (Gautier et al., 2012; Mossadegh-Keller et al., 2017). Within the

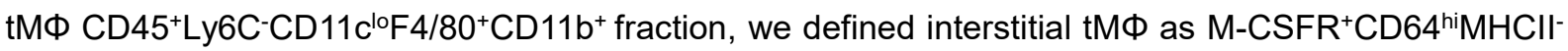
cells and peritubular $\mathrm{TM} \Phi$ as $\mathrm{M}-\mathrm{CSFR}{ }^{\mathrm{lo}} \mathrm{CD} 64^{10} \mathrm{MHCll}^{+}$cells (Figure 3 ).

Here, we describe IF and FACS protocols that will be instrumental to define and isolate these two tMФ populations for further phenotypic and functional characterization.

\section{Materials and Reagents}

1. Pipette tips

2. Microscope slides (SuperFrost Plus, VWR, catalog number: 631-0108)

3. Cover glass (VWR, catalog number: 470820)

4. Hydrophobic pen, Mini PAP Pen (Life Technologies, catalog number: 008877)

5. Simport Scientific Disposable Base Molds (Fisher Scientific, catalog number: 11670990)

6. Eppendorf tube $1.5 \mathrm{ml}$ (Sigma-Aldrich, catalog number: Z606340-1000EA)

7. Sterilin tube (Thermo Fisher Scientific, catalog number: 129A)

8. $5 \mathrm{ml}$ Falcon Polystyrene Round-Bottom Tube (Fisher Scientific, Corning, catalog number: 352008)

9. Filter $50 \mu \mathrm{m}$ Filcon, Sterile, Syringe-type (Becton, catalog number: 340601)

10. Wild-type C57BL/6J mice from Janvier labs

11. CX3CR1GFP/+ mice (Jung et al., 2000)

12. Ice

13. DPBS 1x (Gibco, Life Technologies, catalog number: 14190-094)

14. HBSS 1x (Gibco, Life Technologies, catalog number: 14025-050)

15. BSA (Sigma-Aldrich, catalog number: A2153)

16. Fetal Calf Serum (Biosera, catalog number: FB-1001/500)

17. Saponin (Sigma-Aldrich, catalog number: 47036)

18. Antigenfix (Diapath, catalog number: P0014)

19. Optimum Cutting Temperature (O.C.T.) Tissue-Tek (VWR, catalog number: 256008-930)

20. Isopentane (VWR, catalog number: 24872-323)

21. Antibodies:

a. Anti-CD16/32 (clone 2.4G2, BD Biosciences, catalog number: 553142, working dilution 1/200)

b. Anti-CD45-BV421 (clone 30F11, BD Biosciences, catalog number: 560501, working dilution 1/200)

c. Anti-F4/80-BV785 (clone BM8, BioLegend, catalog number: 123141, working dilution 1/200) 
d. Anti-CD11b-BV605 (clone M1/70, BD Biosciences, catalog number: 563015, working dilution 1/800)

e. Anti-CD64-PerCP/Cy5.5 (clone X54-5/7.1, BioLegend, catalog number: 139308, working dilution 1/300)

f. Anti-CD11C-BV711 (clone N418, BioLegend, catalog number: 117349, working dilution 1/300)

g. Anti-CD115-PE (clone AFS98, eBiosciences, catalog number: 112-1152-82, working dilution $1 / 200)$

h. Anti-Ly6C-APC/Cy7 (clone HK1.4, BioLegend, catalog number: 128026, working dilution 1/300)

i. Anti-I-A/I-E (MHCII)-PE/Cy7 (clone M5/114.15.2, BioLegend, catalog number: 107630, working dilution 1/300)

j. Anti-F4/80-Alexa Fluor647 (clone BM8, BioLegend, catalog number: 123122, working dilution 1/200)

k. Anti-MCII-efluor450 (clone M5/114.15.2, eBiosciences, catalog number: 48-5321-82, working dilution 1/500)

I. Anti-M-CSFR (clone C-20, Santa Cruz, catalog number: sc-692, working dilution 1/800)

m. Anti-rabbit-Alexa Fluor 594 (Jackson Immunoresearch, catalog number: 711-585-152, working dilution 1/500)

22. Prolong Gold antifade reagent (Life Technologies, catalog number: P36930)

23. Zombie Violet fixable live/dead staining (BioLegend, catalog number: BLE423113, working dilution $1 / 1,000$ )

24. $30 \%$ sucrose (Sigma-Aldrich, catalog number: S9378) (see Recipes)

25. Blocking Buffer for IF (see Recipes)

26. Wash Buffer for IF (see Recipes)

27. Prolong containing Sytox blue dye (Life Technologies, catalog number: S34857) (see Recipes)

28. Enzymatic digestion Mix (see Recipes)

a. Collagenase II (Worthington Biochemicals/Serlabo Technologies, catalog number: LS004174, $125 \mathrm{U} / \mathrm{mg}$ dry weight)

b. DNase I (Roche, catalog number: 10104159001)

c. Working enzymatic digestion (500 $\mu / /$ testis sample)

\section{Equipment}

1. Pipettes

2. Microscope slide holder/box (Heathrow Scientific, catalog number: 15994G)

3. Vertical plastic staining rack

4. Dissection scissors (Harvard Apparatus, catalog number: 72-8422)

5. Milligramme balance (Mettler Toledo, catalog number: 30029085) 
6. SnapFrost80 (Alphelys)

7. Cryostat (Leica, model: CM3050S)

8. Confocal microscope (ZEISS, model: LSM 780)

9. 40x/1.4 oil differential interference contrast objective (Plan-Apochromat)

10. FACS LSRII instrument (BD)

11. Thermomixer comfort (Eppendorf, catalog number: 5382000015)

12. Rocking agitator (VWR, catalog number: 444-0756)

13. Centrifuge

14. Vortexer

15. $4{ }^{\circ} \mathrm{C}$ refrigerator

16. $-80^{\circ} \mathrm{C}$ freezer

\section{Software}

1. FlowJo (Version 10.0.8)

2. Image (Version 1.49s, National Institutes of Health)

3. Adobe Illustrator CS6 (Version 16.0.3)

\section{Procedure}

A. Preparing tissue testis sections for IF (Figure 1)

1. Euthanize mice by cervical dislocation.

2. Excise the whole testis organ and place it directly in a Sterilin tube with $1 \mathrm{ml}$ cold Antigenfix. Incubate on a rocking agitator for $3 \mathrm{~h}$ at $4{ }^{\circ} \mathrm{C}$.

3. Wash the fixed testis in cold PBS $1 \mathrm{x}$ at $4{ }^{\circ} \mathrm{C}$ for $20 \mathrm{~min}$.

4. Incubate the testis organ in $5 \mathrm{ml}$ of $30 \%$ sucrose solution overnight at $4{ }^{\circ} \mathrm{C}$, until the testis sinks completely to the bottom of the tube.

5. Transfer the testis into disposable base molds in a coronal plane and place immediately into cold $\left(-80^{\circ} \mathrm{C}\right)$ isopentane inside the SnapFrost80. After $2 \mathrm{~min}$, remove the frozen molds and store at $-80^{\circ} \mathrm{C}$.

6. Section the mouse testis at $20 \mu \mathrm{m}$-thick with the cryostat on SuperFrost slides and store at $-20^{\circ} \mathrm{C}$. 


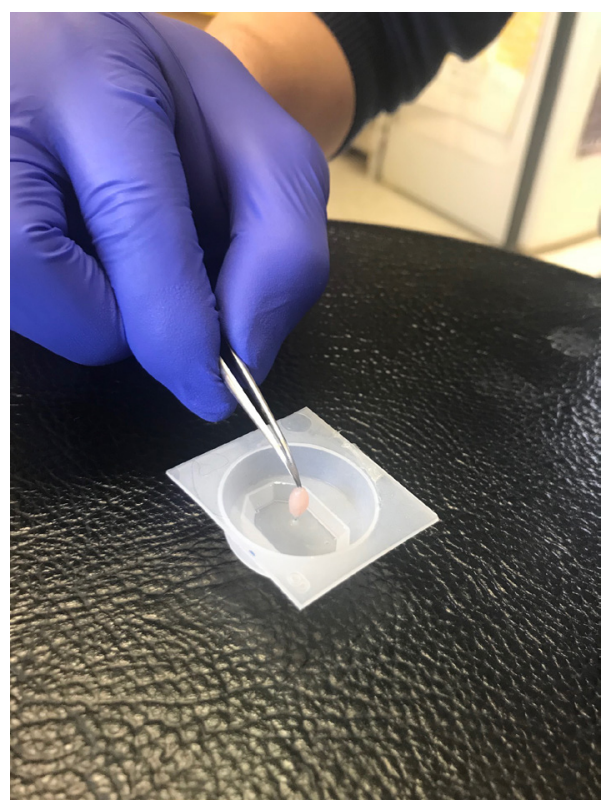

Figure 1. Coronal testis plane preparation. The testis is placed into disposable base molds with OCT in a coronal plane.

B. Immunofluorescence (Figure 2)

1. Place slides in a microscope slide holder/box, delimit the testis section with a hydrophobic pen and add $100 \mu \mathrm{l}$ PBS $1 \mathrm{x}$ per section for $10 \mathrm{~min}$ at room temperature (RT).

2. Incubate in blocking buffer for $1 \mathrm{~h}$ at RT.

3. Wash 3 times with the wash buffer solution for $5 \mathrm{~min}$ at RT by moving the slide rack up and down between each wash.

4. Place wet tissues into the slide holder/box in order to make a humid chamber for the staining.

5. Incubate with antibodies ( $100 \mu \mathrm{l}$ per section) in $\mathrm{PBS} / 0.05 \%$ saponin overnight at $4{ }^{\circ} \mathrm{C}$ at the following dilutions:

a. Anti-F4/80-Alexa Fluor647 dilution 1/200

b. Anti-MCII-efluor450 dilution 1/500

c. Anti-M-CSFR dilution $1 / 800$

6. After three washes using the wash buffer for 5 min each at RT, incubate for $1 \mathrm{~h}$ at RT with antirabbit-Alexa Fluor 594, dilution 1/500, for anti-MCSFR antibody detection.

7. Wash slides three times with $\mathrm{PBS} / 0.05 \%$ saponin for 5 min each at RT by moving the slide rack up and down several times. Place slides on a vertical plastic staining rack, protected from the light, in order to dry the slides.

8. Mount slides in Prolong containing Sytox blue dye (see Recipes). 

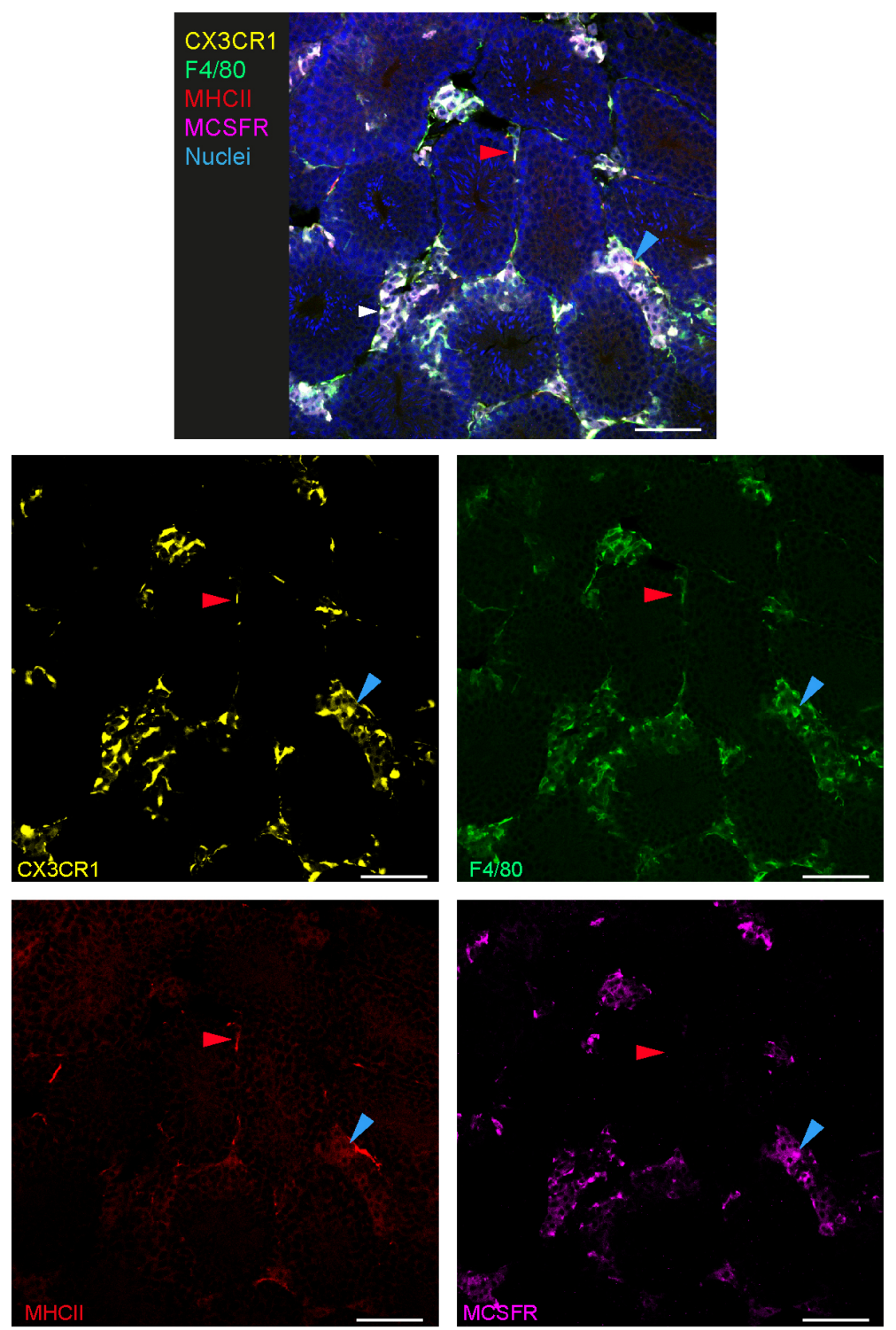

Figure 2. Phenotypic characterization of interstitial and peritubular macrophages by IF. IF imaging of CX3CR1GFP/+ adult mouse testis is revealing morphology, localization, F4/80, MHCII and M-CSFR staining of interstitial and peritubular testicular macrophages. One example of interstitial macrophages is indicated with a blue arrow showing a cell with round morphology and expressing CX3CR1, F4/80 and M-CSFR. One example of peritubular macrophages is indicated with a red arrow showing a cell with elongated morphology and expressing CX3CR1, $\mathrm{F} 4 / 80$ and $\mathrm{MHCII}$. Scale bars $=40 \mu \mathrm{m}$.

C. Fluorescence-Activated Cell Sorting (FACS) (Figure 3)

1. Euthanize mice by cervical dislocation.

2. Collect the whole testis organ and place it directly in a $1.5 \mathrm{ml}$ Eppendorf tube in cold PBS containing $1 \mathrm{mg} / \mathrm{ml}$ Collagenase $11 / 0.15 \mathrm{mg} / \mathrm{ml}$ DNase I. Mechanically dissociate the testis by mincing with scissors directly inside the tube.

3. Place the minced tissue in a thermomixer at $37^{\circ} \mathrm{C}$ for $40 \mathrm{~min}$ by shaking for enzymatic digestion. 
4. Transfer the digested testis through a $50 \mu \mathrm{m}$ filter placed on top of a $5 \mathrm{ml}$ Falcon tube and filter using $1 \mathrm{ml}$ PBS.

5. Centrifuge the digested tissue in a refrigerated centrifuge for $5 \mathrm{~min}$ at $300 \times \mathrm{g}$ at $4{ }^{\circ} \mathrm{C}$.

6. Resuspend the pellet of cells in $200 \mu \mathrm{l}$ of cold PBS and incubate with blocking anti-CD16/32 antibody, dilution $1 / 200$, leaving it on ice for $15 \mathrm{~min}$.

7. Wash in $1 \mathrm{ml}$ cold PBS and centrifuge for $5 \mathrm{~min}$ at $300 \times g$ at $4{ }^{\circ} \mathrm{C}$.

8. Incubate single-cell suspensions in cold PBS and stain for expression of surface antigens for $20 \mathrm{~min}$ on ice. The following antibodies are used in the same mix preparation:
a. Anti-F4/80-BV785 dilution $1 / 200$
b. Anti-CD11b-BV605 dilution $1 / 800$
c. Anti-CD64-PerCP/Cy5.5 dilution 1/300
d. Anti-CD11C-BV711 dilution 1/300
e. Anti-M-CSFR-PE dilution 1/200
f. Anti-Ly6C-APC/Cy7 dilution 1/300
g. Anti-I-A/I-E (MHCII)-PE/Cy7 dilution 1/300
h. Anti-CD45-BV421 dilution 1/200
i. Zombie Violet fixable live/dead cell dye as a viability marker dilution $1 / 1,000$

9. Wash in $1 \mathrm{ml}$ cold PBS and centrifuge for $5 \mathrm{~min}$ at $300 \times \mathrm{g}$ at $4{ }^{\circ} \mathrm{C}$. Take up single cell suspension in $800 \mu \mathrm{l}$ of PBS and pass the solution through a $50 \mu \mathrm{m}$ filter. The sample is ready for the analysis on FACS.
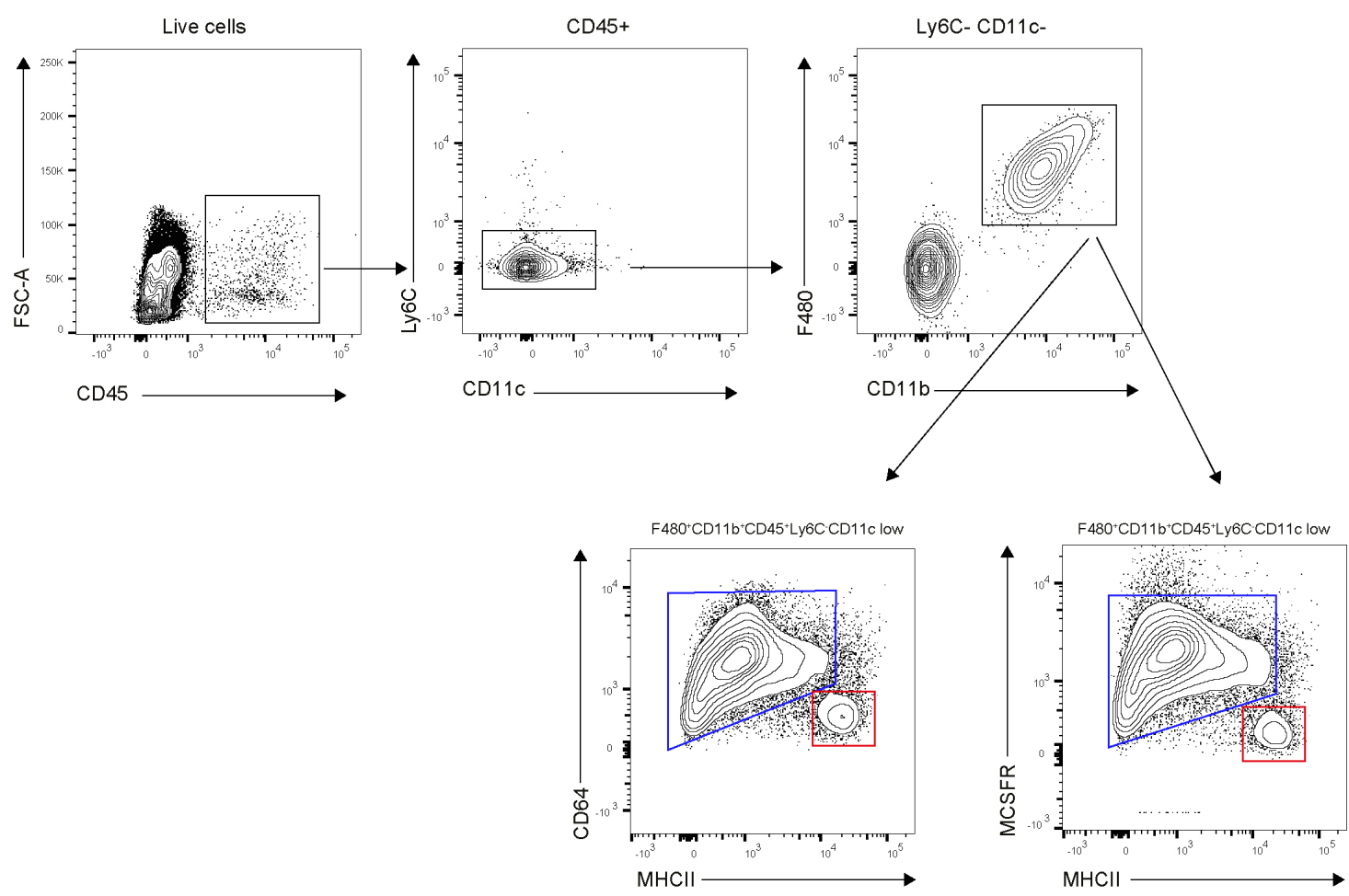

Figure 3. Phenotypic characterization of interstitial and peritubular macrophages by FACS. Gating strategy describing in $C D 45^{+} L y 6 C-C D 11 c^{-} F 4 / 80^{+} C D 11 b^{+}$adult mouse testis fraction, the 
distinction of interstitial $\mathrm{M}-\mathrm{CSFR}{ }^{+} \mathrm{CD} 64^{+} \mathrm{MHCIl}^{-}$and peritubular $\mathrm{M}-\mathrm{CSFR}{ }^{\circ \circ} \mathrm{CD} 64^{10} \mathrm{MHClI}^{+}$population, respectively represented by blue and red gates.

\section{Data analysis}

Confocal microscopy acquisitions were performed on a confocal microscope (LSM780; ZEISS) at room temperature, and slides were imaged with a 40x/1.4 oil differential interference contrast objective (Plan-Apochromat). Different lasers were used ( $405 \mathrm{~nm}, 488 \mathrm{~nm}, 56 \mathrm{~nm}$, and $633 \mathrm{~nm}$ ) to excite the fluorophores (Sytox blue, eFluor 450, Alexa Fluor 488, Alexa Fluor 594, and Alexa Fluor 647). Fluorescence was recorded in individual channels acquired in a sequential mode to avoid cross-talk using a highly sensitive 32-channel gallium arsenide phosphide detector. The pinhole was set to 1 airy unit. Image processing was done with ImageJ (National Institutes of Health). Only a median filter was applied to the images to remove salt and pepper noise.

FACS data were acquired on an LSR II instrument (BD) using violet laser $405 \mathrm{~nm}$, blue laser $488 \mathrm{~nm}$, green laser $561 \mathrm{~nm}$, and red laser $633 \mathrm{~nm}$. FACS data were analyzed using FlowJo software (V10.0.8). The FACS gating strategy to distinguish Interstitial CD64hiMHCII- tMФ and peritubular $\mathrm{CD} 64^{\circ}{ }^{\circ} \mathrm{MHCI}{ }^{+}$tMФ of adult mouse testis and a representative FACS profile is shown in Figure 2. This can be used to quantify the proportion of these two tMФ populations and the details regarding the analysis can be found in the original article (Mossadegh-Keller et al., 2017; e.g., Figures $2 \mathrm{~A}$ and $2 \mathrm{~B}$ ).

\section{$\underline{\text { Recipes }}$}

1. $30 \%$ sucrose

$30 \mathrm{~g}$ sucrose

Bring the volume to $100 \mathrm{ml}$ with PBS

Store at $4{ }^{\circ} \mathrm{C}$

2. Blocking Buffer for IF

$2 \mathrm{~g} \mathrm{BSA}$

$1 \mathrm{ml} \mathrm{FCS}$

$0.1 \mathrm{~g}$ saponin

Bring the volume to $100 \mathrm{ml}$ with PBS

Store at $4{ }^{\circ} \mathrm{C}$

3. Wash Buffer for IF

$0.05 \mathrm{~g}$ saponin

Bring the volume to $100 \mathrm{ml}$ with PBS

Store at $4{ }^{\circ} \mathrm{C}$

4. Prolong containing Sytox blue dye

$1 \mathrm{ml}$ of Prolong without DAPI 
Add $3 \mu \mathrm{l}$ of Sytox blue dye

Vortex 3 min

Add $1 \mathrm{ml}$ Prolong without DAPI

Store at $-20^{\circ} \mathrm{C}$

5. Enzymatic digestion Mix

a. Collagenase $50 \mathrm{mg} / \mathrm{ml}$ stock solution

Resuspend $1 \mathrm{~g}$ of collagenase in $20 \mathrm{ml}$ of HBSS

Make aliquots of $1 \mathrm{ml}$

Store at $-20{ }^{\circ} \mathrm{C}$

b. DNase I $10 \mathrm{mg} / \mathrm{ml}$ stock solution

Resuspend $100 \mathrm{mg}$ of DNase in $10 \mathrm{ml}$ of HBSS

Make aliquots of $500 \mu \mathrm{l}$

Store at $-20^{\circ} \mathrm{C}$

c. Working enzymatic digestion (500 $\mu / /$ testis sample)

$10 \mu \mathrm{l}$ of collagenase stock solution

$7.5 \mu$ l of DNase I stock solution, bring the volume to $500 \mu$ l with PBS

\section{Acknowledgments}

We thanks the Centre d'Immunologie de Marseille-Luminy (CIML) flow cytometry and mouse house facilities for support. We acknowledge the PICSL imaging facility of the CIML (Imaglmm), member of the national infrastructure France-Biolmaging supported by the French National Research Agency (ANR-10-INBS-04).

This work was supported by institutional grants from Institut National de la. Santé et de la Recherche Médicale, Centre National de la Recherche Scientifique, and Aix-Marseille University to the CIML and grants to M.H. Sieweke from the Agence Nationale de la Recherche (ANR-11-BSV30026), Fondation pour la Recherche Médicale (DEQ. 20110421320) a INSERM-Helmholtz cooperation grant and the European Research Council (ERC) under the European Union's Horizon 2020 research and innovation program (grant agreement number 695093 MacAge). M.H. Sieweke has been supported as a BIH-Einstein visiting fellow at the MDC and an Alexander von Humboldt Professor at CRTD/TU Dresden.

\section{Competing interests}

The authors declare no competing financial interests. 


\section{Ethics}

In vivo procedures were performed under specific pathogen-free conditions following protocols approved by the Ethics Committee of Marseille in accordance with institutional, national, and European regulations (approval nos. APAFIS 3292-2015 122109359224 and APAFIS 10545-2017 $071008253541)$.

\section{References}

1. DeFalco, T., Potter, S. J., Williams, A. V., Waller, B., Kan, M. J. and Capel, B. (2015). Macrophages contribute to the spermatogonial niche in the adult testis. Cell Rep 12(7): 11071119.

2. Gautier, E. L., Shay, T., Miller, J., Greter, M., Jakubzick, C., Ivanov, S., Helft, J., Chow, A., Elpek, K. G., Gordonov, S., Mazloom, A. R., Ma'ayan, A., Chua, W. J., Hansen, T. H., Turley, S. J., Merad, M., Randolph, G. J. and Immunological Genome, C. (2012). Gene-expression profiles and transcriptional regulatory pathways that underlie the identity and diversity of mouse tissue macrophages. Nat Immunol 13(11): 1118-1128.

3. Gentek, R., Molawi, K. and Sieweke, M. H. (2014). Tissue macrophage identity and self-renewal. Immunol Rev 262(1): 56-73.

4. Gordon, S. (2002). Pattern recognition receptors: Doubling up for the innate immune response. Cell 111(7): 927-930.

5. Jung, S., Aliberti, J., Graemmel, P., Sunshine, M. J., Kreutzberg, G. W., Sher, A. and Littman, D. R. (2000). Analysis of fractalkine receptor CX(3)CR1 function by targeted deletion and green fluorescent protein reporter gene insertion. Mol Cell Biol 20(11): 4106-4114.

6. Lavin, Y., Winter, D., Blecher-Gonen, R., David, E., Keren-Shaul, H., Merad, M., Jung, S. and Amit, I. (2014). Tissue-resident macrophage enhancer landscapes are shaped by the local microenvironment. Cell 159(6): 1312-1326.

7. Mossadegh-Keller, N. and Sieweke, M. H. (2018). Testicular macrophages: Guardians of fertility. Cell Immunol 330: 120-125.

8. Mossadegh-Keller, N., Gentek, R., Gimenez, G., Bigot, S., Mailfert, S. and Sieweke, M. H. (2017). Developmental origin and maintenance of distinct testicular macrophage populations. $J$ Exp Med 214(10): 2829-2841.

9. Smith, L. B. and Walker, W. H. (2014). The regulation of spermatogenesis by androgens. Semin Cell Dev Biol 30: 2-13.

10. Yona, S., Kim, K. W., Wolf, Y., Mildner, A., Varol, D., Breker, M., Strauss-Ayali, D., Viukov, S., Guilliams, M., Misharin, A., Hume, D. A., Perlman, H., Malissen, B., Zelzer, E. and Jung, S. (2013). Fate mapping reveals origins and dynamics of monocytes and tissue macrophages under homeostasis. Immunity 38(1): 79-91. 